\title{
GENERALIZED MINKOWSKI CONTENT AND THE VIBRATIONS OF FRACTAL DRUMS AND STRINGS
}

\author{
Christina Q. He and Michel L. Lapidus
}

\begin{abstract}
In [La1], the second author has obtained a sharp error estimate for the eigenvalue distribution of the Laplacian on bounded open sets $\Omega \subset R^{n}$ with fractal boundaries (i.e., 'fractal drums'). Further, he and Pomerance [LaPo1,2] studied in detail the case of 'fractal strings' (i.e., $n=1)$ and established in the process some unexpected connections with the Riemann zeta-function $\zeta=\zeta(s)$ in the 'critical strip' $0<\operatorname{Re} s<1$. Later on, still when $n=1$, Lapidus and Maier [LaMa1,2] obtained a new characterization of the Riemann hypothesis by means of an associated inverse spectral problem.

In this paper, we will extend most of these results by using, in particular, the notion of generalized Minkowski content which is defined through some suitable 'gauge functions' other than the power functions. In the situation when the power function is not the natural 'gauge function', this will enable us to obtain more precise estimates, with a broader potential range of applications than in the above papers. Complete proofs of the results announced here will be provided in [HeLa].
\end{abstract}

\section{Introduction}

Let $\Omega$ be a nonempty open set in $\mathbf{R}^{n}(n \geq 1)$, with finite volume and boundary $\Gamma=\partial \Omega$. Consider the following eigenvalue problem:

$$
(P) \quad\left\{\begin{aligned}
-\Delta u & =\lambda u & & \text { in } \Omega \\
u & =0 & & \text { on } \Gamma,
\end{aligned}\right.
$$

where $\Delta=\sum_{k=1}^{n} \partial^{2} / \partial x_{k}^{2}$ denotes the Dirichlet Laplacian on $\Omega$.

As is well known, the spectrum of $(\mathrm{P})$ is discrete and consists of a sequence $\left(\lambda_{k}\right)_{k=1}^{\infty}$ of eigenvalues (with finite multiplicity) written in increasing order according to their multiplicity: $0<\lambda_{1} \leq \lambda_{2} \leq \cdots \leq \lambda_{k} \leq$ $\cdots$, with $\lambda_{k} \rightarrow \infty$ as $k \rightarrow \infty$. Let $N(\lambda)$ denote the eigenvalue counting function of $(\mathrm{P})$; that is, for $\lambda>0, N(\lambda)=\#\left\{k \geq 1: \lambda_{k} \leq \lambda\right\}$.

Received March 14, 1995.

Mathematics Subject Classification. Primary 35P20, 11M06, 58F19. Secondary $28 \mathrm{~A} 75$.

Research partially supported by the National Science Foundation under grant DMS9207098 . 
Next, we recall the standard definitions of the Minkowski dimension and content. (See, e.g., [Fa, La1, Tr].)

Definition 1.1. Given $d>0$, the d-dimensional upper Minkowski content of $\Gamma=\partial \Omega$ is given by

$$
M^{*}(d ; \Gamma)=\limsup _{\epsilon \rightarrow 0^{+}} \epsilon^{-(n-d)}\left|\Gamma_{\epsilon} \cap \Omega\right|_{n},
$$

where $\Gamma_{\epsilon}=\left\{x \in \mathbf{R}^{n} \cap \Omega: d(x, \Gamma)<\epsilon\right\}$. Similarly, we can define the lower Minkowski content $M_{*}(d ; \Gamma)$ by taking the lower limit in (1.1). The Minkowski dimension $D$ of $\Gamma$ is then defined by

$$
D=\inf \left\{d: M^{*}(d ; \Gamma)<\infty\right\}=\sup \left\{d: M^{*}(d ; \Gamma)=\infty\right\} .
$$

Further, we say that $\Gamma$ is Minkowski measurable if

$$
0<M_{*}(d ; \Gamma)=M^{*}(d ; \Gamma)<\infty \text { for some } d>0,
$$

and we then call this common value $M(d ; \Gamma)$ the Minkowski content of $\Gamma$. (In this case, it is clear that $d=D$.)

We say that $\Gamma$ is 'fractal' if $D \in(n-1, n]$, and 'nonfractal' if $D=n-1$.

In [La1, Theorem 2.1], Lapidus proved in particular, that if $\Gamma$ is 'fractal' with $M^{*}(D ; \Gamma)<\infty$, then Weyl's asymptotic law with sharp error term holds:

$$
N(\lambda)=\varphi(\lambda)+O\left(\lambda^{D / 2}\right), \text { as } \lambda \rightarrow \infty,
$$

where $\varphi(\lambda)=(2 \pi)^{-n} \mathcal{B}_{n}|\Omega|_{n}$. Here, $|A|_{n}$ denotes the $n$-dimensional Lebesgue measure of $A \subset \mathbf{R}^{n}$ and $\mathcal{B}_{n}$ is the volume of the unit ball in $\mathbf{R}^{n}$. (For related results, see, e.g., [BrCa, La2] and the references therein.)

In [LaPo1,2], Lapidus and Pomerance obtained the following more precise result in the one-dimensional case (i.e., when $n=1$ ): If $\Omega \subset \mathbf{R}$ has 'fractal' boundary $\Gamma$ which is Minkowski measurable and has Minkowski dimension $D \in(0,1)$, then

$$
N(\lambda)=\varphi(\lambda)-c_{1, D} M(D ; \Gamma) \lambda^{D / 2}+o\left(\lambda^{D / 2}\right), \text { as } \lambda \rightarrow \infty,
$$

where $c_{1, D}$ is given by

$$
c_{1, D}=2^{D-1} \pi^{-D}(1-D)(-\zeta(D)), \varphi(\lambda)=\pi^{-1}|\Omega|_{1} \lambda^{1 / 2}
$$

and $\zeta(s)$ is the Riemann zeta-function. Recall (e.g., from [LaPo2]) that

$$
\zeta(s)=\frac{1}{s-1}+\int_{1}^{\infty}\left([t]^{-s}-t^{-s}\right) d t, \text { for Re } s>0 .
$$

Later on, Lapidus and Maier [LaMa1,2] also examined the corresponding inverse spectral problem. They showed in particular the following 
result: Let $\rho=D+i \nu(0<D<1, \nu \in \mathbf{R})$ be a zero of the Riemann zeta-function. Then there exists an open set $\Omega \subset \mathbf{R}$ of finite length and with boundary $\Gamma$ of Minkowski dimension $D$, such that as $\lambda \rightarrow \infty$, $N(\lambda)=\varphi(\lambda)+C \lambda^{D / 2}+o\left(\lambda^{D / 2}\right)$, for some nonzero constant $C$, but $\Gamma$ is not Minkowski measurable.

The goal of the present paper is to extend these theorems and related results in [LaPo2] by using more general 'gauge functions' than the power function in (1.1). This will significantly broaden the potential range of applications of the above results.

\section{Statement of the main results}

We now give the generalized definition of $h$-Minkowski content which will be used throughout this paper.

Definition 2.1. Let $\Omega \subset \mathbf{R}^{n}$ be an open set with finite volume and boundary $\Gamma=\partial \Omega$. Let $h:(0, \infty) \rightarrow(0, \infty)$ be a nondecreasing function. The upper $h$-Minkowski content of $\Gamma$ is defined by

$$
M^{*}(h ; \Gamma)=\limsup _{\epsilon \rightarrow 0^{+}} \epsilon^{-n} h(\epsilon)\left|\Gamma_{\epsilon} \cap \Omega\right|_{n} .
$$

We define similarly the lower $h$-Minkowski content $M_{*}(h ; \Gamma)$ by taking the lower limit in (2.1). Further, we say that $\Gamma$ is h-Minkowski measurable if $0<M_{*}(h ; \Gamma)=M^{*}(h ; \Gamma)<\infty$, and denote this common value $M(h ; \Gamma)$ the $h$-Minkowski content of $\Gamma$.

Clearly, the standard definition (recalled in Definition 1.1) just corresponds to the case when $h(x)=x^{d}$.

2.1 One-dimensional case $(\mathbf{n}=\mathbf{1})$. In the following, we will extend the results in $[\mathrm{LaPo}]$ and some of the results in [LaMa].

Let $\Omega$ be a nonempty open subset of $\mathbf{R}$ with finite length $|\Omega|_{1}$ and with boundary $\Gamma=\partial \Omega$. We write $\Omega$ as the union of its connected components: $\Omega=\cup_{j=1}^{\infty} I_{j}$, where the open intervals $I_{j}$ are pairwise disjoint and of length $l_{j}$. Since $|\Omega|_{1}=\sum_{j=1}^{\infty} l_{j}<\infty$, we can assume without loss of generality that $l_{1} \geq l_{2} \geq \cdots \geq l_{j} \geq \cdots>0$. Further, we say that $\left(l_{j}\right)_{j=1}^{\infty}$ is the sequence associated with $\Omega$. Then, with $[\gamma]$ denoting the integer part of $\gamma \in \mathbf{R}$, we have

$$
N(\lambda)=\sum_{j=1}^{\infty}\left[l_{j} x\right], \text { where } x:=\frac{\sqrt{\lambda}}{\pi} .
$$

Let us specify the family of gauge functions that will be used in the present case when $n=1$. 
Definition 2.2. Given $d \in(0,1)$, let $G_{d}$ be the class of functions $h$ which satisfy all the following conditions:

$(\mathrm{H} 1) . h:(0, \infty) \rightarrow(0, \infty)$ is a continuous strictly increasing positive function, and $\lim _{x \rightarrow 0^{+}} h(x)=0, \lim _{x \rightarrow \infty} h(x)=\infty, \lim _{x \rightarrow 0^{+}} h(x) / x=\infty$.

(H2). For any $t>0$,

$$
\lim _{x \rightarrow 0^{+}} \frac{h(t x)}{h(x)}=t^{d}
$$

uniformly in $t$ on any compact subset of $(0, \infty)$.

(H3). There exist some constants $\tau \in(0,1), m>0,0<x_{0} \leq 1,0<$ $t_{0} \leq 1$ such that

$$
\frac{h(t x)}{h(x)} \geq m t^{\tau}, \text { for all } 0<x<x_{0}, 0<t<t_{0} .
$$

One can check that the functions

$$
h(x)=\frac{x^{d}}{\left(\ln \left(\frac{1}{x}+1\right)\right)^{a}} \text { and } h(x)=\frac{x^{d}}{\left(\ln \left(\ln \left(\frac{1}{x}+1\right)\right)\right)^{a}}
$$

are in $G_{d}$ for all $d \in(0,1)$ and $a \geq 0$.

Notation. From now on, given $h \in G_{d}$, we will always let

$$
g(x):=h^{-1}(1 / x), \quad f(x):=\frac{1}{h(1 / x)} .
$$

The following two theorems extend [LaPo2, Theorems 2.1 and 2.2].

Theorem 2.3. (a) Let $\left(l_{j}\right)_{j=1}^{\infty}$ be an arbitrary nonincreasing positive sequence such that for some $h \in G_{d}$ and some constant $L>0$, we have

$$
l_{j} \sim \operatorname{Lg}(j), \text { as } j \rightarrow \infty
$$

(i.e., $l_{j} / g(j) \rightarrow L$ as $j \rightarrow \infty$ ). Then

$$
\sum_{j=1}^{\infty}\left[l_{j} x\right]=\left(\sum_{j=1}^{\infty} l_{j}\right) x+\zeta(d) L^{d} f(x)+o(f(x)), \quad \text { as } \quad j \rightarrow \infty
$$

(b) In particular, if the sequence $\left(l_{j}\right)_{j=1}^{\infty}$ associated with $\Omega$ satisfies hypothesis (2.5), then by letting $x=\sqrt{\lambda} / \pi$ in (2.6), we deduce that

$$
N(\lambda)=\varphi(\lambda)+\pi^{-d} \zeta(d) L^{d} f(\sqrt{\lambda})+o(f(\sqrt{\lambda})), \quad \text { as } \lambda \rightarrow \infty,
$$

where $\varphi(\lambda)=\pi^{-1}|\Omega|_{1} \lambda^{1 / 2}=\pi^{-1}\left(\sum_{j=1}^{\infty} l_{j}\right) \lambda^{1 / 2}$. 
Theorem 2.4 (characterization of $h$-Minkowski measurability). The sequence $\left(l_{j}\right)_{j=1}^{\infty}$ associated with $\Omega$ satisfies hypothesis (2.5) if and only if $\Gamma=\partial \Omega$ is h-Minkowski measurable. Further, in this case, the $h$-Minkowski content of $\Gamma$ is given by

$$
M(h ; \Gamma)=\frac{2^{1-d}}{1-d} L^{d} .
$$

By combining Theorems 2.3 and 2.4, we obtain the desired extension of [LaPo2, Corollary 2.3].

Corollary 2.5. Let $\Omega$ be an open set of $\mathbf{R}$ with finite length such that $\Gamma=\partial \Omega$ is $h$-Minkowski measurable for some $h \in G_{d}$. Then we have

$$
N(\lambda)=\varphi(\lambda)-c_{1, d} M(h ; \Gamma) f(\sqrt{\lambda})+o(f(\sqrt{\lambda})), \text { as } \lambda \rightarrow \infty,
$$

where $c_{1, d}=2^{-(1-d)} \pi^{-d}(1-d)(-\zeta(d)) .\left(\right.$ Note that $c_{1, d}>0$.)

The following theorem characterizes the situation when we obtain sharp remainder estimates. It provides an extension of [LaPo2, Theorem 2.4].

Theorem 2.6. Let $\Omega$ be an open subset of $\mathbf{R}$ with finite length. Let $\left(l_{j}\right)_{j=1}^{\infty}$ be the associated sequence, and let $h \in G_{d}$ for some $d \in(0,1)$. Then the following assertions are equivalent:

(1) $l_{j} \asymp g(j)$, as $j \rightarrow \infty$;

(2) $0<M_{*}(h ; \Gamma) \leq M^{*}(h ; \Gamma)<\infty$;

(3) $\sum_{j=1}^{\infty}\left\{l_{j} x\right\} \asymp f(x)$, as $x \rightarrow \infty$;

(4) $\varphi(\lambda)-N(\lambda) \asymp f(\sqrt{\lambda})$, as $\lambda \rightarrow \infty$.

Here, $\{\gamma\}=\gamma-[\gamma]$ denotes the fractional part of the real number $\gamma$. Further, ' $v(x) \asymp w(x)$ as $x \rightarrow a$ ' means that there exist positive constants $c_{1}, c_{2}$ such that $c_{1} v(x) \leq w(x) \leq c_{2} v(x)$, for all $x$ in some neighborhood of $a$.

We also obtain the following partial extension of [LaMa2, Theorem 3.2].

Theorem 2.7. Suppose $h \in G_{d} \quad$ is a differentiable function with $x h^{\prime}(x) / h(x) \geq \mu>0$, for all $x>0$ and some constant $\mu$. Furthermore, let $\rho=d+i \nu(0<d<1, \nu \in \mathbf{R})$ be a zero of the Riemann zeta-function. Then we can construct an open set $\Omega \subset \mathbf{R}$ such that $\Gamma=\partial \Omega$ is not $h$-Minkowski measurable, but

$$
N(\lambda)=\varphi(\lambda)+c f(\sqrt{\lambda})+o(f(\sqrt{\lambda}), \quad \text { as } \lambda \rightarrow \infty,
$$

for some constant $c<0$. Moreover, $0<M_{*}(h ; \Gamma) \leq M^{*}(h ; \Gamma)<\infty$. 
2.2 Higher dimensional case. Let $n$ be any integer $\geq 2$. We will make the following assumption on the gauge function $h$ in this case:

$(\mathrm{C} 1) . h:(0, \infty) \rightarrow(0, \infty)$ is a positive nondecreasing function. Moreover, $\lim _{x \rightarrow 0^{+}} h(x)=0, \lim _{x \rightarrow \infty} h(x)=\infty$.

(C2). There exist constants $k_{1}, k_{2}$, with $2^{n-1}<k_{1} \leq k_{2} \leq 2^{n}$, such that $k_{1} h(x) \leq h(2 x) \leq k_{2} h(x)$, for all $x$ small.

(C3). $h(x) \leq c x^{n-1}$ for some constant $c>0$ and for all $x$ small.

(C4). $h(x) / x^{n} \rightarrow 0$ as $x \rightarrow \infty$.

For example, if $h$ is given by (2.3) with $d \in(n-1, n)$ and $a \geq 0$, then it satisfies $(\mathrm{C} 1)-(\mathrm{C} 4)$.

We can now state our extension of [La1, Theorem 2.1] to this setting.

Theorem 2.8 (error estimate). Suppose that $h$ satisfies $(\mathrm{C} 1)-(\mathrm{C} 4)$. Let $\Omega \subset \mathbf{R}^{n}$ be an open set with finite volume and with boundary $\Gamma=\partial \Omega$ of finite upper $h$-Minkowski content; i.e., $M^{*}(h ; \Gamma)<\infty$. Then we have

$$
N(\lambda)=\varphi(\lambda)+O(f(\sqrt{\lambda})) \text {, as } \lambda \rightarrow \infty .
$$

We will see in Example 4.1 that the above error estimate in (2.10) is sharp in general.

Full statements and proofs of our results announced here are provided in $[\mathrm{HeLa}]$.

Remark 2.9. After this work was completed, we have learned that a result analogous to Theorem 2.8-generalizing [La1] Theorem 2.1 along the lines of [La1] Remark 2.4(e), p. 481 for gauge functions of the type of Eq. (2.3) — was obtained independently by A. M. Caetano in [Ce2], $\S 5$.

\section{Sketch of the proof of Theorem 2.3}

The following preliminary results are used in the proof of Theorem 3.2 below as well as throughout the proofs of other theorems in $\S 2.1$.

Proposition 3.1. Suppose $h \in G_{d}$ for some $d \in(0,1)$. Then:

(i) Let $\left(a_{j}\right)_{j=1}^{\infty}$ in $(0, \infty)$ be bounded away from 0 and $\infty$, and let $\left(b_{j}\right)_{j=1}^{\infty}$ in $(0, \infty)$ be such that $b_{j} \rightarrow 0$ as $j \rightarrow \infty$. Then we have

$$
\lim _{j \rightarrow \infty} \frac{h\left(a_{j} b_{j}\right)}{a_{j}^{d} h\left(b_{j}\right)}=1
$$

(ii) Suppose $\left(a_{j}\right)_{j=1}^{\infty}$ is as in (i) and $\lim _{j \rightarrow \infty} b_{j}=\infty$. Then we have

$$
\lim _{j \rightarrow \infty} \frac{g\left(a_{j} b_{j}\right)}{a_{j}^{-1 / d} g\left(b_{j}\right)}=1 .
$$


(iii)

$$
\lim _{x \rightarrow \infty} \frac{\int_{x}^{\infty} g(u) d u}{x g(x)}=\frac{d}{1-d} .
$$

We now provide a sketch of the proof of Theorem 2.3, which we restate as follows.

Theorem 3.2. Suppose $l_{1} \geq l_{2} \geq \cdots>0$ and $l_{j} \sim \operatorname{Lg}(j)$ as $j \rightarrow \infty$, for some constant $L>0$ and $h \in G_{d}$. Let $\delta(x)=\left(\sum_{j=1}^{\infty} l_{j}\right) x-\sum_{j=1}^{\infty}\left[l_{j} x\right]=$ $\sum_{j=1}^{\infty}\left\{l_{j} x\right\}$. Then

$$
\delta(x) \sim-\zeta(d) L^{d} f(x), \quad \text { as } x \rightarrow \infty .
$$

Proof. Let $J(\epsilon)=\max \left\{j \geq 1: l_{j} \geq \epsilon\right\}$. Since $\frac{l_{j}}{g(j)}=\frac{l_{j}}{h^{-1}\left(\frac{1}{j}\right)} \rightarrow$ $L \quad$ as $j \rightarrow \infty$, by Proposition 3.1(i), we have $\frac{h\left(l_{j}\right)}{h\left(h^{-1}(1 / j)\right)}=\frac{h\left(l_{j}\right)}{1 / j}=$ $j h\left(l_{j}\right) \rightarrow L^{d}$, as $j \rightarrow \infty$. Thus $J(\epsilon) h\left(l_{J(\epsilon)}\right) \rightarrow L^{d}$ as $\epsilon \rightarrow 0^{+}$. We note that $\frac{l_{J(\epsilon)}}{\epsilon} \rightarrow 1$ as $\epsilon \rightarrow 0^{+}$, and so, by Proposition 3.1(i) again, $h\left(l_{J(\epsilon)}\right) / h(\epsilon) \rightarrow 1$ as $\epsilon \rightarrow 0^{+}$. Hence

$$
J(\epsilon) h(\epsilon)=J(\epsilon) h\left(l_{J(\epsilon)}\right) \frac{h(\epsilon)}{h\left(l_{J(\epsilon)}\right)} \rightarrow L^{d} \cdot 1=L^{d}, \text { as } \epsilon \rightarrow 0^{+}
$$

Let $k \geq 2$ be an arbitrary fixed integer. We obtain as in [LaPo2, Eq. (4.16)], upon applying Abel's summation formula, that

$$
\begin{aligned}
\delta(x) & =x \sum_{j>J(1 / x)} l_{j}+\sum_{j \leq J(k / x)}\left\{l_{j} x\right\}+\sum_{q=2}^{k} \sum_{j=J(q / x)+1}^{J\left(\frac{q-1}{x}\right)}\left\{l_{j} x\right\} \\
& =: A+B+C,
\end{aligned}
$$

where

$$
\begin{aligned}
A & :=x \sum_{j>J(k / x)} l_{j}, \quad B:=k J\left(\frac{k}{x}\right)-\sum_{q=1}^{k-1} J\left(\frac{q}{x}\right), \\
C & :=\sum_{j \leq J(k / x)}\left(\left\{l_{j} x\right\}-1\right) .
\end{aligned}
$$

We now estimate, for fixed $k$, each of these terms as $x \rightarrow \infty$.

Since $-1 \leq\left\{l_{j} x\right\}-1<0$, we have $-J(k / x) \leq C<0$; thus as $x \rightarrow \infty$ :

$$
0 \leq-L^{-d} h\left(\frac{1}{x}\right) C \leq L^{-d} \frac{h(1 / x)}{h(k / x)} J\left(\frac{k}{x}\right) h\left(\frac{k}{x}\right) \rightarrow L^{-d} \cdot k^{-d} \cdot L^{d}=k^{-d} .
$$


Next, we deduce from the definition of $B$ and (3.1) that

$$
L^{-d} h\left(\frac{1}{x}\right) B \rightarrow k^{1-d}-\sum_{q=1}^{k-1} q^{-d}, \text { as } x \rightarrow \infty .
$$

Further, we claim that

$$
L^{-d} h\left(\frac{1}{x}\right) A \rightarrow k^{1-d} \frac{d}{1-d}, \text { as } x \rightarrow \infty .
$$

Indeed, since $\alpha_{j}=l_{j} / g(j) \rightarrow L$ as $j \rightarrow \infty$ and $J(\epsilon) h(\epsilon) \rightarrow L^{d}$ as $\epsilon \rightarrow 0^{+}$, we see that for each $\epsilon>0$, there is some $x_{0}>0$ such that for all $x \geq x_{0}$, we have $\alpha_{j} \in(L-\epsilon, L+\epsilon)$, for all $j>J\left(\frac{k}{x}\right)$. Thus, for all $x \geq x_{0}$,

$$
\begin{aligned}
L^{-d} h\left(\frac{1}{x}\right) A \leq & L^{-d}(L+\epsilon) x h\left(\frac{1}{x}\right) \int_{J(k / x)}^{\infty} g(t) d t \\
= & (L+\epsilon) L^{-d} x\left(\frac{h(1 / x)}{h(k / x)}\right)\left(h\left(\frac{k}{x}\right) J\left(\frac{k}{x}\right)\right) \\
& \cdot\left(\frac{g(J(k / x))}{g\left(\frac{1}{h(k / x)}\right)}\right) g\left(\frac{1}{h(k / x)}\right) \frac{\int_{J(k / x)}^{\infty} g(t) d t}{J(k / x) g(J(k / x))} \\
= & (L+\epsilon) L^{-d} x \cdot k^{-d} \cdot L^{d} \cdot L^{-1} \cdot \frac{k}{x} \cdot \frac{d}{1-d}(1+o(1)) \\
= & \left(1+\frac{\epsilon}{L}\right) k^{1-d} \frac{d}{1-d}(1+o(1)), \text { as } x \rightarrow \infty,
\end{aligned}
$$

where we have used (H2), (3.1), as well as Proposition 3.1 (ii) and (iii) in the second equality. Similarly, we can prove that

$$
L^{-d} h\left(\frac{1}{x}\right) A \geq\left(1-\frac{\epsilon}{L}\right) k^{1-d} \frac{d}{1-d}(1+o(1)), \text { as } x \rightarrow \infty .
$$

Since $\epsilon$ can be chosen arbitrarily small, we see by combining (3.5) and (3.6) that (3.4) holds.

Thus, for fixed $k$, by (3.3) and (3.4), we have as $x \rightarrow \infty$,

$$
L^{-d} h\left(\frac{1}{x}\right)(A+B) \rightarrow k^{1-d} \frac{d}{1-d}+k^{1-d}-\sum_{q=1}^{k-1} q^{-d}=w_{k}(d)+\frac{1}{1-d},
$$

where $w_{k}(s):=\int_{1}^{k}\left(t^{-s}-[t]^{-s}\right) d t\left(=-\frac{1}{1-s}+\frac{1}{1-s} k^{1-s}-\sum_{q=1}^{k-1} q^{-s}\right)$. It follows from (1.3) that $w_{k}(d)+\frac{1}{1-d} \rightarrow-\zeta(d)$, as $k \rightarrow \infty$.

Putting (3.2) and (3.7) together, we deduce that $\lim _{x \rightarrow \infty} L^{-d} h\left(\frac{1}{x}\right) \delta(x)$ $=-\zeta(d)$. This yields Theorem 3.2, and hence Theorem 2.3. 


\section{Example}

In this section, we illustrate our results by an example which will show in particular that our estimate in Theorem 2.8 is in general best possible. Example 4.1 In [Ce1], Caetano constructed the following bounded open set $\Omega$ in $\mathbf{R}^{2}$ (see Fig. 1), which extends Example 5.1 in [La1]. Let $a(x)=$ $x^{-1} \ln x$ and let

$$
\Omega:=\cup_{j=3}^{\infty}(a(j+1), a(j)) \times\left(-\frac{1}{2}, \frac{1}{2}\right) .
$$

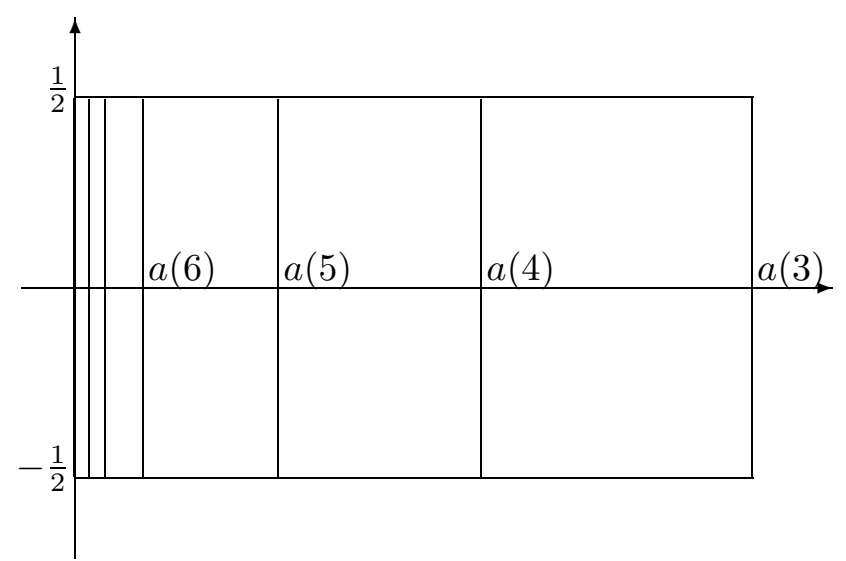

Fig. 1 The open set $\Omega$ : fractal comb

Let

$$
h(x)=\frac{x^{3 / 2}}{\left(\ln \left(x^{-1}+1\right)\right)^{1 / 2}} .
$$

It can be shown that $h$ satisfies $(\mathrm{C} 1)-(\mathrm{C} 4)$ and $0<M_{*}(h ; \Gamma) \leq M^{*}(h ; \Gamma)$ $<\infty$. Thus, by Theorem 2.8 above, we have as $\lambda \rightarrow \infty$,

$$
N(\lambda)-\varphi(\lambda)=O(f(\sqrt{\lambda}))=O\left(\lambda^{3 / 4}(\ln \lambda)^{1 / 2}\right) .
$$

We actually obtain a sharp estimate in this case. Indeed, by Corollary 2.15 in [Ce1], we have as $\lambda \rightarrow \infty$,

$$
N(\lambda)-\varphi(\lambda) \asymp \lambda^{3 / 4}(\ln \lambda)^{1 / 2} .
$$

Similar results, involving a broad range of gauge functions, hold for a large family of the open sets constructed in [Ce1]. Furthermore, as in [La1], Examples 5.1 and $5.1^{\prime}$, such examples can clearly be constructed in any dimension $n \geq 1$. In particular, when $n=1$, they can be used to illustrate 
many of our results in $\S 2.1$. Other examples, related to Theorems 2.3, 2.6 and 2.7 , are provided in [HeLa].

\section{References}

[BrCa] J. Brossard and R. Carmona, Can one hear the dimension of a fractal? Comm. Math. Phys. 104 (1986), 103-122.

[Ce1] A. M. Caetano, Some domains where the eigenvalues of the Dirichlet Laplacian have non-power second term asymptotic estimates, J. London Math. Soc. (2) 43 (1991), 431-450.

[Ce2] _ _ On the search for the asymptotic behavior of the eigenvalues of the Dirichlet Laplacian for bounded irregular domains, Internat. J. Scientific Computing \& Modelling (to appear).

[Fa] K. Falconer, Fractal geometry, Wiley, Chichester, 1990.

[HeLa] C. Q. He and M. L. Lapidus, Generalized Minkowski content, spectrum of fractal drums, fractal strings and the Riemann zeta-function, preprint, IHES/M/95/47, Institut des Hautes Etudes Scientifiques, Bures-Sur-Yvette, France, to appear in Memoirs Amer. Math. Soc..

[La1] M. L. Lapidus, Fractal drum, inverse spectral problems for elliptic operators and a partial resolution of the Weyl-Berry conjecture, Trans. Amer. Math. Soc. 325 (1991), 465-529.

[La2] _ _ Vibrations of fractal drums, the Riemann hypothesis, waves in fractal media, and the Weyl-Berry conjecture, Pitman Research Notes in Mathematics Series 289, Longman, London, 1993, pp. 126-209.

[LaMa1] M. L. Lapidus and H. Maier, Hypothèse de Riemann, cordes fractales vibrantes et conjecture de Weyl-Berry modifiée, C. R. Acad. Sci. Paris Sér. I Math. 313 (1991), 19-24.

[LaMa2] _ The Riemann hypothesis and inverse spectral problems for fractal strings, J. London Math. Soc. (2) No.1, 52 (1995), 15-34.

[LaPo1] M. L. Lapidus and C. Pomerance, Fonction zêta de Riemann et conjecture de Weyl-Berry pour les tambours fractals, C. R. Acad. Sci. Paris Sér. I Math. 310 (1990), 343-348.

[LaPo2] __ The Riemann zeta-function and the one-dimensional Weyl-Berry conjecture for fractal drums, Proc. London. Math. Soc. (3) 66 (1993), 41-69.

[Tr] C. Tricot, Two definitions of fractal dimension, Math. Proc. Cambridge Philos. Soc. 91 (1988), 57-74.

Department of Mathematics, Sproul Hall, University of California RiverSIDE, CA 92521-0135, USA

E-mail address: che@math.ucr.edu, lapidus@math.ucr.edu 